\title{
Synthetic cannabinoid, bonzai, intoxication via a narghile
}

\begin{abstract}
Synthetic cannabinoids are becoming increasingly popular with adolescents as an abused drug in many countries. Synthetic cannabinoids, referred to as Bonzai in Turkey, are relatively new recreational drugs of abuse. The most common route of these drugs is smoking. In this case report, I firstly reported a synthetic cannabinoid, bonzai, intoxication that was occurred through water pipe, narghile, using.
\end{abstract}

Volume 4 Issue 4 - 2016

\author{
Ahmet Eroglu \\ Karadeniz Technical University, Anesthesiology and Reanimation, \\ Trabzon, Turkey
Correspondence: Ahmet Eroglu, Karadeniz Technical University, Anesthesiology and Reanimation, Trabzon, Turkey, Email aheroglu@hotmail.com

Received: February 05, 2016 | Published: April 05, 2016

\section{Introduction}

A hookah is also known as a water pipe, narghile, or by other names. It is a single or multi-stemmed instrument for vaporizing and smoking flavoured tobacco. Narghile is a type of smoking pipe used in eastern countries in which the smoke is filtered through a water pipe before reaching the inhaler pipe. In the Middle East and Arab world, people smoke water pipe as part of their culture and traditions. Health risks of smoking hookah include exposure to toxic chemicals that are not filtered out by the waterand risk of infectious disease when hookahs are shared. ${ }^{1,2}$

Synthetic cannabinoids are becoming increasingly popular with adolescents as an abused drug in many countries. Synthetic cannabinoids, referred to as Bonzai in Turkey, are relatively new recreational drugs of abuse. ${ }^{3}$ The most common route of these drugs is smoking. In this case report, I firstly reported a synthetic cannabinoid, bonzai, intoxication that was occurred through water pipe, narghile, using.

\section{Case report}

A 25-year-old man was brought to emergency department by his friends after smoking tobacco and bonzai through a narghile in a café. When he was admitted by the emergency department, he had nausea and vomiting, and aspiration of gastric contain. He was unconscious and his Glasgow Coma score was reported as 8 . His oxygen saturation was $78 \%$ on room air. Clinical examination and his chest $\mathrm{x}$-ray revealed aspiration pneumonia. Because of his aspiration pneumonia and respiratory insufficiency, he was intubated and mechanical ventilation was started. And then he was transferred to the intensive care unit. Antibiotics, mechanical ventilation and other intensive care therapies were administered in the intensive care unit. On the third day, he had a normal blood gas analysis, sufficient spontaneous respiration, a better chest $\mathrm{x}$-ray, and then intubation tube was removed and the weaning from the ventilation was successful. On the seventh day, he was discharged from the hospital with normal function.
To use a narghile to smoke synthetic cannabinoid is very interesting route. I would like to attract attention that synthetic cannabinoids, especially bonzai, are potentially harmful drugs of abuse, and can even cause lethal effects. Adolescents should be carefully while using narghile in a cafe. The written consent has been obtained from the patient.

\section{Acknowledgments}

None.

\section{Conflicts of interest}

Author declare that there is no conflict of interest.

\section{Funding}

None.

\section{References}

1. WHO Study Group on Tobacco Product Regulation. Advisory note: waterpipe tobacco smoking:health effects, research needs and recommended actions by regulator. 2nd ed. World Health Organization, Geneva, USA; 2015

2. Akl EA, Gaddam S, Gunukula, SK, et al. The effects of waterpipe tobacco smoking on health outcomes:a systematic review. Int $J$ Epidemiol. 2010;39(3):834-857.

3. Besli GE, Ikiz MA, Yildirim S, et al. Synthetic Cannabinoid Abuse in Adolescents: A Case Series. J Emerg Med. 2015;49(5):644-650. 